\title{
Le Fonds d'exploration minière du Nunavik
} Promouvoir le potentiel minéral du Nunavik en partenariat avec les Inuits

\section{The Nunavik Mineral Exploration Fund: Promoting the mineral potential of Nunavik in partnership with the Inuit Los Fondos de explotación minera del Nunavik : Promover el potencial minero de Nunavik en asociación con los Inuits}

\author{
Jean-Marc Séguin
}

Volume 40, numéro 3, 2010

URI : https://id.erudit.org/iderudit/1009368ar

DOI : https://doi.org/10.7202/1009368ar

Aller au sommaire du numéro

Éditeur(s)

Recherches amérindiennes au Québec

ISSN

0318-4137 (imprimé)

1923-5151 (numérique)

Découvrir la revue

Citer cet article

Séguin, J.-M. (2010). Le Fonds d'exploration minière du Nunavik : promouvoir le potentiel minéral du Nunavik en partenariat avec les Inuits. Recherches amérindiennes au Québec, 40(3), 53-58. https://doi.org/10.7202/1009368ar

\section{Résumé de l'article}

Le Fonds d'exploration minière du Nunavik (FEMN) a vu le jour en 1998. Cette organisation à but non lucratif est issue d'une entente entre le ministère des Ressources naturelles du Québec, l'Administration régionale Kativik et le Conseil régional de développement Kativik. Depuis sa mise sur pied, le FEMN voit à ce que son mandat et ses objectifs soient atteints à travers les différentes activités qui comprennent : 1 ) la sensibilisation des communautés inuits au développement des ressources minérales et à la promotion du potentiel minéral du territoire ; 2) la formation et l'assistance technique aux prospecteurs inuits ; 3) la réalisation de projets d'exploration minérale propres au FEMN en partenariat avec l'industrie minière ; finalement, 4) la mise en place d'éléments favorisant l'émergence d'un entrepreneuriat inuit dans le domaine de l'industrie minière. Après plus de dix années d'existence, le FEMN a su former une expertise et une main-d'oeuvre locales non seulement disposées à répondre aux besoins des compagnies opérant sur le territoire, mais également prêtes à participer activement au développement des ressources minérales du Nunavik.
Tous droits réservés @ Recherches amérindiennes au Québec, 2010
Ce document est protégé par la loi sur le droit d'auteur. L'utilisation des services d'Érudit (y compris la reproduction) est assujettie à sa politique d'utilisation que vous pouvez consulter en ligne.

https://apropos.erudit.org/fr/usagers/politique-dutilisation/ 


\section{Le Fonds d'exploration minière du Nunavik}

Promouvoir le potentiel minéral du Nunavik

en partenariat avec les Inuits

\section{Jean-Marc Séguin}

Fonds d'exploration minière du Nunavik, Kuujjuaq

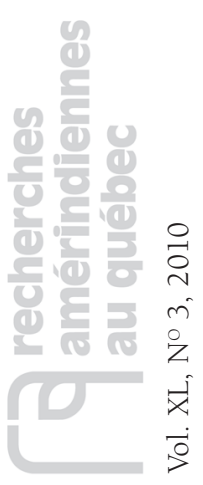

L E GOUVERNEMENT DU QUÉBEC encourage la participation des communautés autochtones du Moyen et Grand Nord au développement des ressources minérales de leurs régions. Une des approches privilégiées consiste à favoriser la création de fonds d'exploration minière dans les communautés autochtones afin de mettre en œuvre la promotion du potentiel minéral, la sensibilisation à l'exploration minière et la formation de la main-d'œuvre, tout comme les activités de prospection et d'exploration. De l'aide technique et financière devient également accessible aux prospecteurs autochtones, et des initiatives d'aide à la création de sociétés d'exploration minière sont mises de l'avant dans le but, pour ce qui est du Grand Nord, de stimuler l'intérêt des communautés inuites en favorisant un développement minier compatible avec leurs valeurs et intégré au milieu social et culturel de la région. De cette façon, les communautés sont encouragées à participer activement à la mise en valeur des ressources minérales du territoire, bénéficiant subséquemment des retombées économiques directes qu'une telle industrie peut générer.

La création de fonds miniers autochtones, dont le Fonds d'exploration minière du Nunavik (FEMN) créé en 1998 de concert avec des organismes régionaux du Nunavik tels que l'Administration régionale Kativik (ARK), le Conseil régional de développement Kativik (CRDK) et la Société Makivik (Makivik), devient un outil de développement en préparation aux initiatives gouvernementales touchant le domaine minier. Des initiatives gouvernementales, viennent appuyer le développement durable des ressources minérales du Nord québécois notamment la Stratégie minérale du Québec lancée en 2009, et, plus récemment, le Plan Nord propose un ambitieux plan de développement tous azimuts, dont le développement minier.

Le Nunavik a accueilli positivement la venue du FEMN. Cet organisme à but non lucratif a tôt fait de consacrer ses efforts à la mise en place d'un plan d'action comprenant : un programme de formation en prospection minérale auprès des communautés inuites; une campagne d'information auprès des leaders du Nunavik au sujet de l'activité minière présente sur le territoire; et l'élaboration de projets d'exploration en vue de nouvelles découvertes minérales en faisant de l'embauche locale une priorité. 


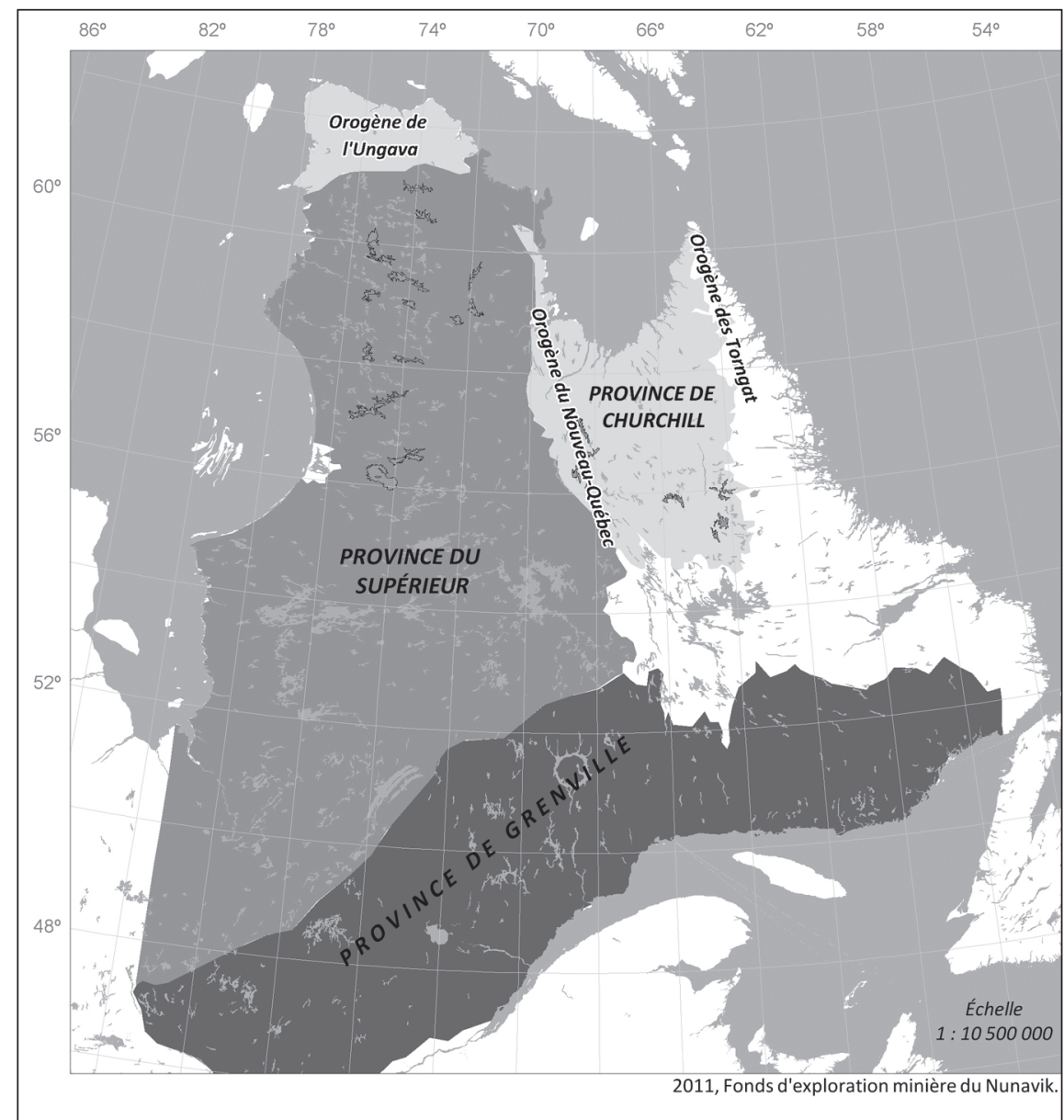

Figure 1

Carte tectonique générale du Québec

\section{Contexte géologiQue et minéral du NunaViK}

Le vaste territoire du Nunavik se situe dans la partie la plus septentrionale du Québec et constitue un terreau riche en ressources naturelles. Outre l'exploitation de minerai de nickel et de cuivre en cours, d'autres gisements importants sont identifiés et répartis sur l'ensemble de la région. Ainsi, des gisements de nickel-cuivre additionnels sont sur le point d'être exploités dans la zone de la ceinture de Raglan, d'importants dépôts de minerai de fer sont maintenant délimités le long de l'axe QuaqtaqSchefferville, un fort potentiel de gisements d'éléments des terres rares est également reconnu à proximité de la frontière du Labrador, des cibles d'exploration polymétalliques sont identifiées dans la région de la côte d'Hudson et de plus, un fort potentiel uranifère existe dans le secteur des monts Torngat.

Le Nunavik possède donc un potentiel minéral immense qui demeure peu exploité malgré les nombreux efforts réalisés en ce domaine depuis plus d'un siècle. En effet, depuis la venue de ces premiers explorateurs dont les contributions géoscientifiques sont encore aujourd'hui pertinentes, un nombre restreint de projets miniers, tant au stade exploratoire que de développement, voient le jour. Laccessibilité limitée ainsi que le climat particulier et rigoureux limitent depuis toujours les levés géoscientifiques et les campagnes d'exploration à la recherche de métaux et minéraux industriels sur le territoire. Il n'en demeure pas moins que plusieurs minéralisations et gîtes minéraux furent mis au jour au fil des années et que de volumineux dépôts de minerai de classe mondiale sont aujourd'hui répertoriés. En effet, au fur et à mesure que les données géoscientifiques étaient recueillies par les nombreux programmes de cartographie et de prospection minérale, l'état des connaissances et un portrait plus précis de la géologie du Nunavik et de son potentiel économique se sont dessinés au terme d'une incessante compilation des travaux effectués dans la région.

À cet égard, il est utile de rappeler que la région est principalement composée de grands assemblages de roches archéennes (>2,5 Ga) à paléoprotérozoïques (entre 2,2 et 1,7 Ga) qui témoignent d'une évolution géologique et tectonique complexe sur une période de plus d'un milliard d'années. La géologie du Nunavik est principalement subdivisée en deux importantes provinces géologiques, soit : a) la province du Supérieur, et b) la province de Churchill. La province du Supérieur constitue un craton archéen occupant le coeur du Bouclier canadien alors que la province de Churchill consiste en un ensemble de blocs cratoniques archéens à paléoprotérozoïques bordés à l'ouest par l'Orogène du Nouveau-Québec et son prolongement au nord, l'Orogène de l'Ungava, et à l'est par l'Orogène des Torngat (fig. 1). Les deux provinces géologiques, du Supérieur et de Churchill, viennent se buter, au sud, sur la province d'âge précambrien de Grenville.

Entre 1997 et 2003, le ministère des Ressources naturelles du Québec a entrepris un ambitieux programme de cartographie géologique dans le nord du Québec, le programme Grand Nord. Cette acquisition de données géoscientifiques dans la Province géologique du Supérieur a contribué de façon importante à la mise à jour de la carte 
géologique de la région du Nunavik et de ses ressources minérales.

Le programme Grand Nord (Simard et al. 2008), échelonné sur cinq années à partir de 1997, est donc venu mettre au jour de nouvelles indications de ressources minérales et ainsi fournir de nouvelles cibles d'exploration à l'industrie minière. Les travaux de ce programme d'acquisition de connaissance se sont principalement concentrés dans la partie nord-est de la province géologique du Supérieur (voir fig. 1). Cette zone d'intérêt, située entièrement au nord du $55^{\mathrm{e}}$ parallèle et comprenant une superficie de près de $350,000 \mathrm{~km}^{2}$, se trouve par le fait même incluse dans le territoire du Nunavik. Des investissements de l'ordre de 26 millions de dollars sont consacrés à cette acquisition de données géoscientifiques, et jusqu'à présent 170 indices minéralisés ont été observés et répartis selon 21 différents types de gîtes minéraux dans la partie nord-est de la Province du Supérieur (Simard et al. 2008) [voir fig. 1]. Des travaux de même envergure, amorcés dès 1970, ont également répertorié près de 400 gîtes minéraux, également selon 21 types de minéralisations, à l'intérieur de la

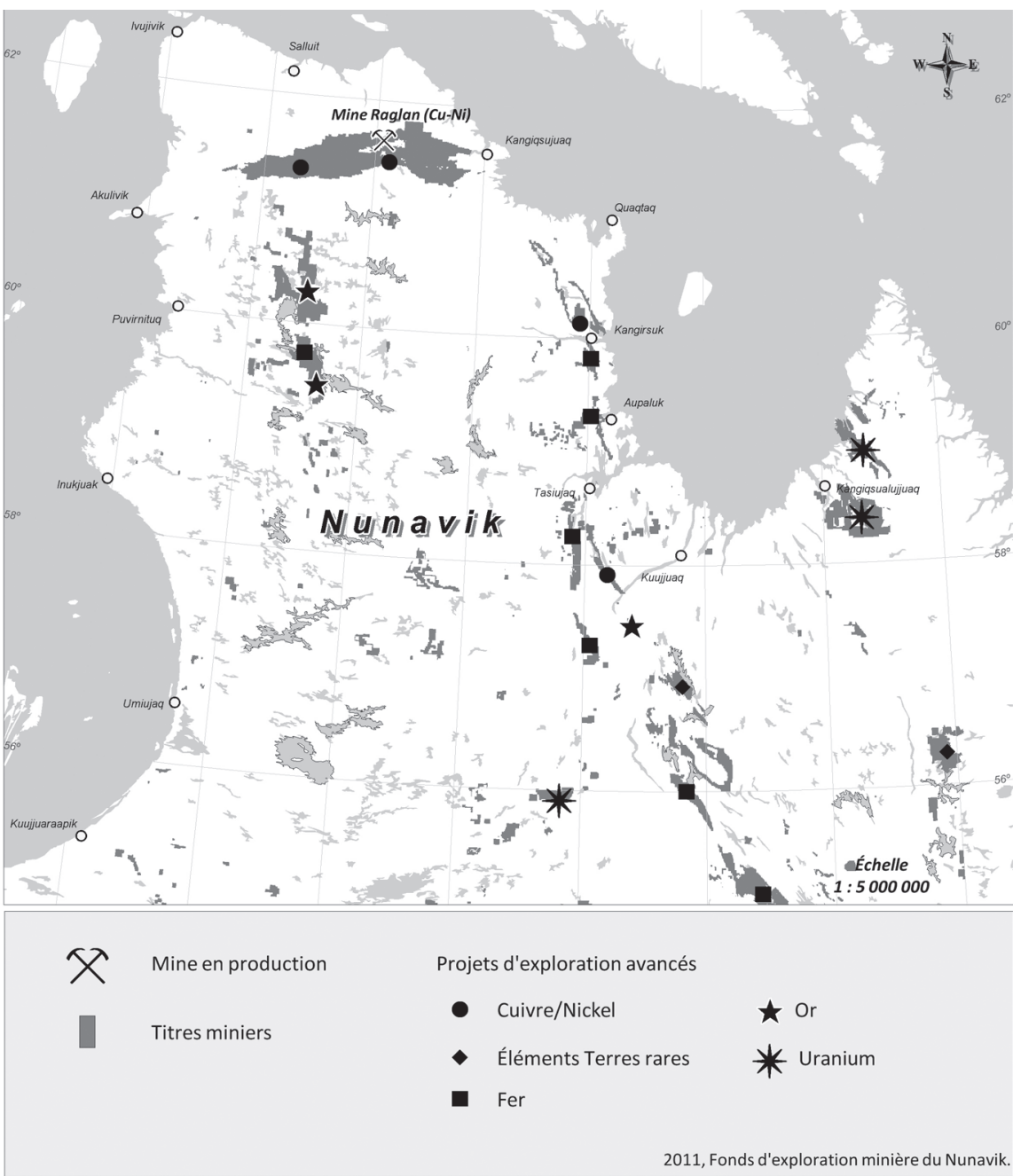

Figure 2

Carte des projets miniers sur le territoire du Nunavik région de l'Orogène du NouveauQuébec (Clark et Wares 2004). De son côté, la région de l'Orogène de l'Ungava compte à elle seule plus d'une centaine de gîtes aurifères et de métaux usuels. Cette impressionnante quantité de gisements et indices minéralisés sur l'ensemble du territoire témoigne clairement de la diversité et de l'importance du potentiel minéral dont jouit le Nunavik.

Éventuellement, l'exploitation de certains de ces gisements ouvrirait la voie et encouragerait la réalisation d'autres projets d'exploration minière pouvant générer une activité minière renouvelée sur le territoire. À titre d'exemple, la mine Raglan, située dans l'Orogène de l'Ungava (fig. 2), a amorcé la production de minerai de nickel-cuivre en 1998.

Le nord du Québec a été peu exploré jusqu'à présent. Nous voulons accroître nos connaissances afin d'inciter les entreprises à y faire de l'exploration minière. La Société minière Raglan vient d'inaugurer une importante mine de nickel près de Purtuniq. Nous pensons qu'il peut y avoir d'autres belles découvertes. (Denise Carrier-Perreault, ministre déléguée aux Mines, gouvernement du Québec, 1997)

Jusqu'à ce jour, plus de neuf millions de tonnes de minerai ont été extraites de la mine Raglan à raison de plus d'un million de tonnes par année. Les réserves des ressources de la mine Raglan sont renouvelées annuellement et sont maintenant évaluées à plus de seize millions de tonnes, cette évaluation demeurant d'ailleurs non définitive et à majorer. À ce rythme, on estime que l'exploitation de telles ressources s'échelonnera sur près d'un siècle. Conséquemment, toute la région de l'Orogène de l'Ungava est maintenant jalonnée par plusieurs autres compagnies minières. D'autres importants dépôts de cuivre-nickel faisant l'objet de travaux avancés sont en opération dans cette région (voir fig. 2).

Plus au sud, un considérable gisement de fer est localisé dans l'Orogène du Nouveau-Québec. Une première 
évaluation estime les ressources à plus de six milliards de tonnes de minerai. Un autre gisement, cette fois d'éléments des terres rares (ETR), fut relevé dans la province de Churchill où plus de cent millions de tonnes de minerai sont estimés. Ce dépôt minéral particulier vient positionner la région comme un incontournable acteur dans des filières spécifiques liées aux technologies vertes, entre autres la catalyse, les énergies renouvelables, le stockage d'énergie et les superalliages. Le potentiel multiéléments du territoire lui assure donc une consolidation et une diversification de l'exploration et de la mise en valeur des ressources minérales avec une forte possibilité de développer un créneau innovateur.

\section{Création du Fonds d'exploration minière DU NunAviK}

Depuis la mise en production de la mine Raglan et la conduite d'importants travaux d'acquisition de connaissances sur le territoire, le Nunavik vit des années déterminantes quant au développement de ses ressources minérales. Une condition essentielle cependant: tout développement doit nécessairement inclure les communautés inuites qui habitent et utilisent le territoire, et ce, en concertation avec les administrateurs et les organismes régionaux. De plus, ce développement doit se concrétiser tout en respectant les droits exclusifs d'activités traditionnelles et territoriaux des communautés et à cet égard la Convention de la Baie James et du Nord québécois comporte des dispositions claires qui encadrent le développement minier sur le territoire du Nunavik.

En septembre 1997, une entente portant sur le développement des ressources minérales du Nunavik a été conclue entre le gouvernement du Québec, l'Administration régionale Kativik et le Conseil régional de développement Kativik. Cette entente prévoyait la création d'un fonds d'exploration minière dirigé par un conseil d'administration formé de représentants d'organismes du milieu. Laide financière accordée à l'organisme à but non lucratif s'inscrit dans le cadre du Programme de développement de l'entrepreneuriat minier autochtone dans les régions du Moyen et du Grand Nord.

Le Fonds d'exploration minière du Nunavik (FEMN) a donc vu le jour en 1998. Une première rencontre entre les différents intervenants, en février 1998, a permis de définir les objectifs et mandats du FEMN, de dresser un premier plan d'action et des prévisions budgétaires. Cette entente assure le versement d'une aide financière annuelle de 300000 \$ afin de couvrir les dépenses reliées à la conduite des activités du FEMN. Les organisations régionales, notamment l'ARK et plus récemment Makivik, organisation à but non lucratif représentant les Inuits du Nunavik au terme de la Convention de la Baie James et du Nord québécois (CBJNQ), participent au fonctionnement du FEMN notamment par l'entremise de programmes d'aide financière à la formation et à l'embauche.

Les bureaux du FEMN sont situés à Kuujjuaq et font maintenant partie du Centre de recherche du Nunavik. Son conseil d'administration est composé de six directeurs, nommés par les différents organismes régionaux : l'ARK, Makivik et l'Association des corporations foncières du Nunavik (ACFN); de plus, un représentant du MRNF y est membre d'office.

\section{Mandat et objectifs du FEMN}

\section{Sensibilisation et PROMOtion}

Dès sa création, le FEMN se voit confier par le gouvernement du Québec le mandat de sensibiliser la communauté du Nunavik au potentiel minéral du territoire et aux occasions d'affaires qui en découlent. Le FEMN s'assure de conduire des activités de promotion auprès de la population et des organismes régionaux, activités qui comprennent la production et la diffusion de documents sur les campagnes de l'industrie minière en cours tant à l'échelle régionale que locale. Le FEMN participe également à l'organisation de sessions d'information et tente de tenir à jour un portrait des projets d'exploration minière en opération sur le territoire. Par exemple, en 2008 , le FEMN rassemblait les maires et les administrateurs du Nunavik ainsi que les promoteurs de l'industrie minière à Kuujjuaq lors du Kuujjuaq Mining Workshop. Cette session d'information visait à présenter, par le biais de conférences, les différents projets miniers avancés (en voie de passer au stade d'exploitation de ressources) dans la région et à encourager et faciliter les échanges entre les promoteurs de l'industrie et les représentants des organismes régionaux et locaux, ces derniers étant fort concernés par les activités d'exploration minière conduites sur leur territoire, les problématiques qu'elles peuvent soulever, les retombées qui leur sont associées et les répercussions éventuelles.

De plus le FEMN présente un rapport d'activités lors des rencontres générales annuelles de l'ARK et de l'ACFN et participe également à des colloques et congrès d'ordre régional, provincial et international afin d'encourager les compagnies d'exploration à participer au développement du Nunavik.

Conscient de la valeur de l'utilisation du territoire pour les Inuits et de l'exercice de leurs activités traditionnelles, le FEMN organise également, dans le cadre d'activités de sensibilisation, un «concours de roches » qui récompense les nouvelles découvertes minérales faites par la population du Nunavik. Le taux de participation augmente d'années en années, et un grand nombre de 
personnes soumettent au FEMN des échantillons de roche amassés sur le territoire. Au cours des dernières années, quelques indices minéralisés furent ainsi mis au jour par l'entremise de ce concours.

\section{FORMATION DE PROSPECTEURS INUITS}

Une des activités les plus réussies du FEMN est sûrement la formation en prospection minérale qui est proposée aux communautés. En fait, l'organisation visite chaque année deux communautés et invite les participants intéressés à suivre une formation de prospecteurs, d'échantillonneurs pour les levés de géochimie, d'aides géologues, d'aides géophysiciens. Durant ces sessions, le FEMN voit à ce que chaque participant soit adéquatement équipé en

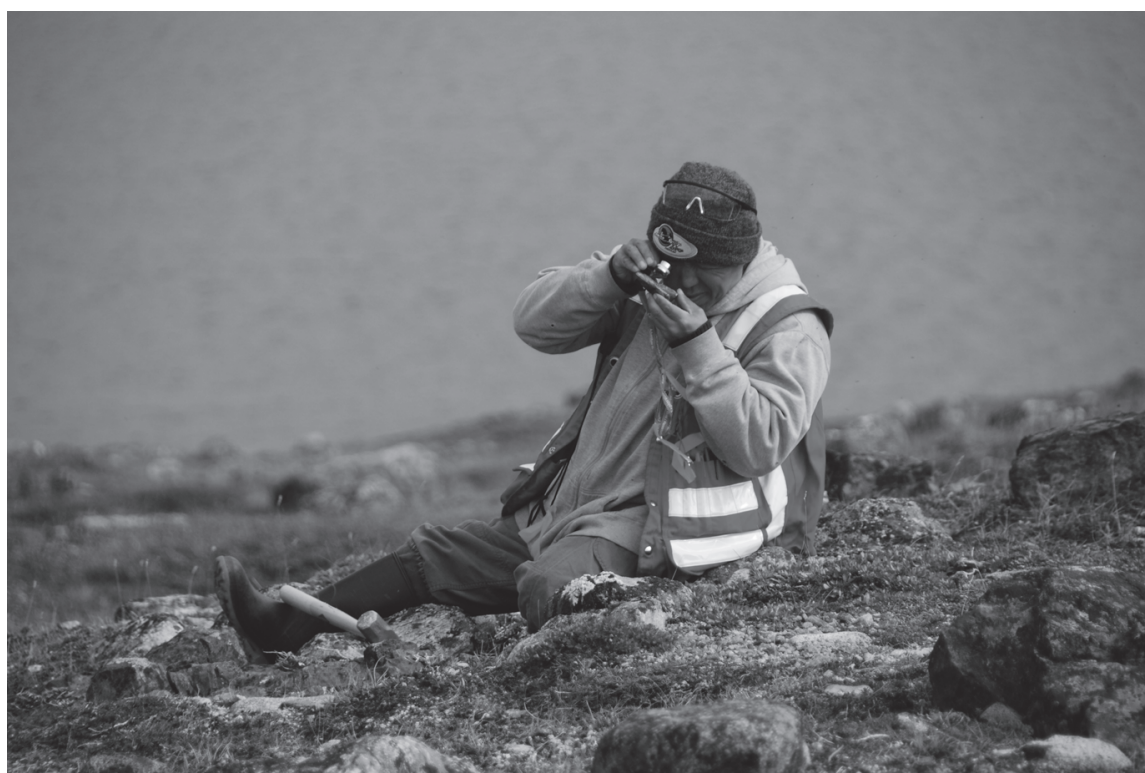

Figure 3

Prospecteur inuit recueillant des observations sur le terrain (Photo de Jean-François Martin, 2009) fournissant le matériel nécessaire afin de participer à la formation. Les sessions sont données directement sur le terrain sur une période de une à deux semaines pendant lesquelles les participants sont informés des concepts théoriques propres à la géologie, à l'identification de roches et minéraux, aux techniques d'échantillonnage, à l'utilisation d'instruments géophysiques et enfin, à la production d'un rapport concis après un levé de prospection. Une fois les formations terminées, les participants ont acquis les connaissances de base en prospection minérale et possèdent les outils afin de se lancer dans des programmes de prospection minérale de façon tout à fait autonome. Ceux-ci voient également leurs noms inscrits sur une liste de références, ce qui augmente leur possibilité d'œuvrer avec les compagnies d'exploration minière qui opèrent dans la région. Depuis 1998, le FEMN a visité en au moins une occasion chacune des quatorze communautés sur le territoire, de même que la communauté de la nation naskapie de Kawawachikamach, afin d'offrir de telles formations. Jusqu'à présent, plus de 160 personnes ont suivi la formation. Bon nombre d'entre elles ont par la suite trouvé un emploi dans des projets réalisés sur le territoire par des compagnies d'exploration. Lindustrie active dans la région a tout à gagner en intégrant le plus possible les communautés au sein de ses projets d'exploration et de développement pour favoriser l'emploi local. Cette intégration accroît le sentiment d'appartenance, l'acceptation sociale des projets et crée une plus grande harmonie entre les communautés et l'industrie.

En lien avec les formations, le FEMN agit également comme guichet unique de soutien aux activités de prospection réalisées par les prospecteurs autonomes. Le FEMN assure un encadrement technique et professionnel adéquat et assiste les prospecteurs dans la réalisation de leur projet. Un programme d'aide financière directe aux prospecteurs pour des projets individuels est aussi offert. Cet appui financier, qui peut atteindre $30000 \$$ par prospecteur par année, est octroyé pour des dépenses liées aux travaux de terrain (location d'équipement, transport, analyses géochimiques, contrats de service, frais de subsistance et autres) et vise à encourager la mise en ouvre de projets de prospection minérale par des prospecteurs inuits autonomes. Depuis la mise sur pied de ce programme d'aide aux prospecteurs autonomes, trente-six projets de prospection minérale ont été réalisés par trente-trois prospecteurs autonomes provenant de huit communautés différentes.

\section{EXPLORATION DES RESSOURCES MINÉRALES DU TERRITOIRE}

L'entente sur le développement des ressources minérales du Nunavik de 1997 prévoit l'allocation de fonds pour les dépenses engagées par le FEMN dans la réalisation de travaux d'exploration minière (géologie, géochimie, géophysique, prospection, échantillonnage, analyse, forage et autres travaux connexes). Ces travaux peuvent être réalisés soit sur des terrains où le FEMN possède des titres miniers, soit sur des cibles minérales potentielles. Les frais de main-d'œuvre pour l'embauche de prospecteurs autochtones dans le cadre de projets de prospection réalisés par le FEMN ou par une entreprise réalisant des activités d'exploration minière sur le territoire de ce dernier, sont également admissibles dans le cadre de l'entente. Le 
FEMN peut aussi participer, avec des entreprises d'exploration minière, à des projets conjoints menés sur le territoire dans le cadre d'ententes de partenariat. Depuis les dix dernières années, le FEMN a mené plus de quarantecinq projets d'exploration, dont une dizaine en partenariat avec l'industrie, dans différents contextes géologiques favorables à la découverte d'indices minéralisés. Ces différents travaux d'échantillonnage sont distribués sur l'ensemble du territoire (fig. 3) et ont conduit à la découverte de pas moins de quarante-six indices minéralisés dans la région, confirmant une fois de plus l'important potentiel minéral multi-élément du Nunavik.

De plus, au cours de chacun de ses travaux exploratoires, l'organisation s'est engagée à faire appel à la maind'œuvre locale et a continuellement donné la priorité à l'embauche de prospecteurs inuits. De cette façon, le FEMN contribue à développer une expertise locale, à permettre une mise à jour continue des connaissances géoscientifiques chez les prospecteurs qui œeuvrent dans des contextes géologiques variés, et concrétise des occasions d'emploi faisant suite aux activités de formation. Le FEMN a donc pu compter sur l'assistance d'un total de quatre-vingt-treize prospecteurs inuits pour l'accomplissement de tous ses travaux d'exploration minérale depuis 1998.

À un autre niveau d'appui à l'exploration minérale, le FEMN offre des services techniques et professionnels à des entreprises d'exploration minière ouvrant sur le territoire: ces offres de services permettent, elles aussi, l'embauche saisonnière de prospecteurs locaux et l'optimisation de l'expertise locale. Aujourd'hui, les compagnies désireuses de développer des projets sur le territoire tout en favorisant la main-d'œuvre locale, n'hésitent plus à contacter le FEMN afin de travailler conjointement avec les communautés. Lindustrie étant disposée à collaborer avec le FEMN, celui-ci arrive à développer un créneau spécifique pour la prospection minérale au Nunavik. Tous les intervenants intéressés dans le développement des ressources minérales peuvent mener à bien des projets d'exploration dans un cadre qui facilite la consultation, la communication d'information et de préoccupations éventuelles, mais surtout la participation des communautés touchées par ces projets.

\section{Création de sociétés publiques d'exploration MINIĖRE INUITES}

Afin de favoriser la création de sociétés d'exploration minière par des promoteurs autochtones, une disposition de l'entente sur le développement des ressources minérales du Nunavik prévoit également que le FEMN peut financer des projets d'exploration réalisés par des entreprises autochtones. Des fonds sont prévus pour couvrir diverses dépenses liées au démarrage d'une entreprise.
Laide accordée par FEMN ne peut excéder 75 \% du coût total d'un projet, jusqu'à un maximum de $50000 \$$. Jusqu'à ce jour aucune compagnie d'exploration minière inuite ne s'est prévalue de ces dispositions.

\section{Conclusion}

Le Fonds d'exploration minière du Nunavik a vu le jour dans la foulée d'un ambitieux programme d'acquisition de connaissances géoscientifiques sur le territoire du Nunavik créé par le gouvernement québécois. Le FEMN est devenu depuis 1998 un outil de développement fort utile aux communautés inuites et il bénéficie d'un support sans équivoque de la part des organisations régionales du Nunavik. Par l'entremise d'activités de promotion et de sensibilisation mais surtout par ses sessions de formation en prospection minérale conjuguées à la réalisation de projets d'exploration en partenariat avec l'industrie, le FEMN contribue à l'augmentation tangible de la participation des communautés au développement minéral en cours sur le territoire. De cette façon, les Inuits peuvent participer activement au développement des ressources minérales tout en acquérant des connaissances et en bénéficiant de retombées économiques immédiates.

Par l'entremise de son engagement dans le milieu et des partenariats réalisés au cours des dernières années, le FEMN a pu développer un créneau bien spécifique dans l'offre de services à l'industrie minière désireuse de venir œuvrer dans la région. Dans la foulée de la Stratégie minérale du Québec et de l'élaboration du Plan Nord, les bases sont jetées afin de permettre la création de compagnies d'exploration minérale inuites. À l'aube de l'élaboration d'un plan de développement stratégique minéral propre au Nunavik, le présent contexte est favorable à l'établissement des éléments fondamentaux d'un développement et d'une gestion durables des ressources minérales du territoire. Ces éléments comprendront en premier lieu la participation active des communautés et le respect, dans leur intégrité, des droits et intérêts reconnus afin d'arriver à la réalisation d'une croissance économique à long terme, d'une autonomie et, enfin, à la prospérité des Inuits.

\section{Ouvrages cités}

CLARK, Thomas, et Robert WARES, 2004 : Synthèse lithotectonique et métallogénique de l'Orogène du Nouveau-Québec (Fosse du Labrador). Ministère des Ressources naturelles et de la Faune, MM 2004-01, Québec.

SIMARD, M., J.-Y. LABBÉ, C. MAURICE, P. LACOSTE, A. LECLAIR et M. BOILY, 2008 : Synthèse du nord-est de la province du Supérieur. Ministère des Ressources naturelles et de la Faune, NM 2008-02, Québec. 